\title{
Hybrid genetic algorithm-based on optimization of multi-echelon inventory structure design
} \author{
Yang $\mathrm{Yu}^{1, \mathrm{a}}$, Zhangen Wang
Xueying $\mathrm{Li}^{1, \mathrm{f}}$, Qiang
Xuang
${ }^{1, \mathrm{c}}$, Wei Yang \\ ${ }^{1}$ School of mechanical engineering, Shandong University, Jinan 250061, China \\ ${ }^{2}$ Shangdong Sihua Environmemt Protect \& Energy-saving Engineering Co.,Ltd \\ ezhenzhengyuyang@163.com, ${ }^{\mathrm{b}}$ zhangenwang@sina.com, c zhangqiangd@163.com, \\ dyming921@163.com, eseki8827@gmail.com, ${ }^{\mathrm{f}}$ dsnowdsw@gmail.com
}

Keywords: Hybrid genetic algorithm; multi-echelon inventory structure

\begin{abstract}
Take inventory and delivery time of raw materials and finished products in the product life cycle from upstream business to the downstream business as the main variable,Integrated cost factors of multiple suppliers, individual manufacturers, multiple distribution center and multiple retailers under the environment of supply chain environment, build Longitudinal multi-level inventory and distribution model (IMLIDM). It uses the improved hybrid simulation genetic algorithm (GSA) optimize the model, instance operation results show that optimization effect of this algorithm is better than Integrated Product and Process Development (IPPD).
\end{abstract}

\section{Introduction}

The first some of literature about inventory and distribution mode were mainly concentrated in the separate for supplier or retailer to determine an optimal solution in order to achieve the lowest cost or profit biggest goal, For example the first proposed Economic order quantity (EOQ) [1], however this solution cannot let buyers and sellers reached their satisfactory results at the same time.

However, with the increasingly furious marketing competition, more and more companies are beginning to realize that they must establish long-term strategic partnership with the rest of the enterprise in the same supply chain, can they meet the needs of the market, some researchers began to develop extensive research on this problem. Through the stock institute established model, we can see that, one the one hand is through the integrated procurement - production (IPP) to establish(Golhar and Sarker[2], Jamal and Sarker[3]), On the other hand is integrated vendor - buyer (IVB) to establish (Nanda and Nam [4], [5] Goyal Goyal and Nebebe [6]).

At present in the process based on the supply chain inventory and distribution mode optimization research, there is no research and analysis on a product characteristics, and failed to consider the customer's actual demand of final consumption goods, most take retail end demand as continuous stability. So from the point view of integrated supply chain, Analysis and research on the optimization of supply chain inventory and distribution mode, be provided with important theoretical significance and realistic significance.

\section{Establishing model}

\section{Symbol Definition.}

The definition of $\mathrm{s}$ is a collection of suppliers $s=1,2, \cdots s$.p represents the manufacturers $d=1,2, \cdots d, \mathrm{~d}$ is the distribution center's collection. $\mathrm{r}$ is a collection of retailers $r=1,2, \cdots r$.

The constant parameters are defined as follows: $m$ - Producer production rate; $L$ - Customer demand lead time; ${ }^{L_{r}}$ - Retailers demand lead time; ${ }^{\lambda_{r i}}$ - The customer demand rate of retailer i; ${ }^{\lambda_{d i}}$ The distribution center i demand rate; ${ }^{\lambda_{p i}}$ - Manufacturers rate of the demand for raw materials; $h_{r i}$ 
Retailer i is the unit inventory holding costs; ${ }^{h_{d i}}$ - The distribution center i unit inventory holding costs; ${ }$ - Per unit of product manufacturer's inventory holding costs; ${ }{ }_{p i}$ - Supplier i's unit inventory holding costs; $A_{r i}$ - Retailer i the unit ordering cost; ${ }_{d i}$ - The distribution center i the unit ordering cost; $^{A_{p i}}$ - Manufacturer of raw materials needed i the unit ordering cost; ${ }^{C_{p}}$ - Manufacturer of the unit production preparation costs; $\pi_{r i}$ - Retailer i unit shortage cost; ${ }^{d i}$ - The distribution center i the unit shortage cost; ${ }^{\pi_{p i}}$ - Manufacturer of raw materials needed i the unit shortage cost; ${ }^{C R_{i}}$ - Raw material $i$ in the consumption rate of the manufacturer;

The variable parameters are defined as follows: $Q_{r}^{i}$ - Retailer i order quantities; $Q_{d}^{i}$ - The distribution center i order quantities; $Q_{p}^{i}$ - Manufacturer order quantities of raw material i; $b_{r i}$ - Retailer i out of stock amount; $b_{d i}$ - The stock amount of the distribution center $\mathrm{i} ;{ }^{p i}$ - the amount of out of stock - manufacturer of raw materials needed; $f_{r i}$ - Order of retailer $\mathrm{i}$ is frequency; $f_{d i}$ - The distribution center $\mathrm{i}$ order frequency; $f_{p i}$ - Manufacturer demand for raw material $\mathrm{i}$ is the frequency; ${ }^{T_{s i}}$ - Supplier i replenishment cycle ${ }^{T_{p}^{i}}$ - The manufacturer of the replenishment cycle of raw material $\mathrm{i} ;{ }^{T_{p}}$ - The manufacturer's production cycle; ${ }^{T_{d}^{i}}$ - The distribution center i replenishment cycle; ${ }^{T}{ }_{r i}$ - Retailer i's replenishment cycle; ${ }^{\hat{T}_{r i}}$ - Out of stock of retailer i in time; ${ }^{T_{s i}^{p}}$ - Raw material supplier i replenishment cycle i at the manufacturer can still consume time;

\section{Cost Assessment.}

\section{1) retailer costs}

$$
\begin{aligned}
& f\left(k ; \lambda_{r i} L\right)=\frac{\left(\lambda_{r i} L\right)^{k}}{k !} e^{-\lambda_{r i} L} \quad \lambda_{r i}>0, \quad k=0,1, \cdots \\
& F\left(k ; \lambda_{r i} L\right)=\sum_{i=k}^{\infty} f\left(k ; \lambda_{r i} L\right)=\sum_{i=k}^{\infty} \frac{\left(\lambda_{r i} L\right)^{k}}{k !} e^{-\lambda_{r i} L} \\
& I_{i}^{e}\left(\sigma_{r i} ; \lambda_{r i}\right)=\sum_{k=0}^{\sigma_{i}}\left(\sigma_{r i}-k\right) f\left(k ; \lambda_{r i} L\right)=\sigma_{r i} F\left(\sigma_{r i} ; \lambda_{r i} L\right)-\sum_{k=0}^{\sigma_{i}} k f\left(k ; \lambda_{r i} L\right) \\
& Q_{r}^{i}=x_{r i} f_{r i}=\sigma_{r i}+\sum_{k=\sigma_{i}+1}^{+\infty}\left(k-\sigma_{r i}\right) f\left(k ; \lambda_{i} L\right)=\lambda_{i}+I_{i}^{e}\left(\sigma_{r i} ; \lambda_{r i} L\right) \\
& \bar{I}_{r i}=\left[\frac{Q_{r}^{i}\left(Q_{r}^{i}+1\right)}{2 \lambda_{r i}}+\frac{Q_{r}^{i} I_{i}^{e}\left(\sigma_{r i}, \lambda_{r i}\right)}{\lambda_{r i}}\right] f_{r i}+\frac{Q_{r}^{i}}{\lambda_{r i}} b_{r i} \\
& b_{r i}=\lambda_{r i} \hat{T}_{r i} f_{r i}=\lambda_{r i} f_{r i}\left(L F\left(k, \lambda_{r i} L\right)-\frac{k}{\lambda_{r i}} F\left(k+1, \lambda_{r i} L\right)\right) \\
& C_{r i}=h_{i r} \bar{I}_{r i}+s_{i r} x_{i} f_{r i}+b_{r i} \pi_{r i} \\
& C_{r}=\sum_{i=1}^{r} C_{r i}=\sum_{i=1}^{r} h_{i r} \bar{I}_{r i}+s_{r i} x_{r i} f_{r i}+b_{r i} \pi_{r i}
\end{aligned}
$$

\section{2) allocation center cost}

$$
\begin{aligned}
Q_{d}^{i} & =\sum_{i=d}^{d_{1}} Q_{r}^{i}=\sum_{i=1}^{d_{1}} \lambda_{i}+I_{i}^{e}\left(\sigma_{r i} ; \lambda_{r i} L\right) \\
Q_{d} & =\sum_{i=1}^{d} Q_{d}^{i}=\sum_{i=1}^{d} \sum_{j=1}^{r} \lambda_{r i}+I_{i}^{e}\left(\sigma_{r i} ; \lambda_{r i} L\right)
\end{aligned}
$$


$C_{d}=\sum_{d=1}^{d} A_{d i} Q_{d i}$

\section{3) production cost}

$$
\begin{aligned}
& I_{s i}^{p}=\frac{1}{2 T_{s}^{i}}\left(\frac{T_{s}^{i}}{k T_{p}}-n_{s i}\right) Q_{p}^{i} k T_{p} \\
& \bar{I}_{p}=\frac{\sum T W I(\alpha, \beta, \gamma, \cdots, \delta)}{T_{p}}=\sum_{d=1}^{d} \frac{1}{T_{p}}\left[\frac{Q_{d}^{i}\left(\sum_{d=1}^{D} \lambda_{d i}\right) f_{d i}}{2 \lambda_{d i}}+\frac{T_{p} Q_{d}^{i}}{2}\left(1-\sum_{d=1}^{D} \lambda_{d i}\right)\left(f_{d i}-1\right)\right] \\
& C_{p}=\bar{I}_{p}\left(h_{p}+c_{p}\right)+\sum_{i=1}^{s} I_{s i}^{p} h_{p i}+A_{p}^{i}\left(f_{s i}-\lambda_{s i}\right)
\end{aligned}
$$

\section{4) supplier cost}

$$
\begin{aligned}
& \bar{I}_{s i}=\frac{1}{T_{s}^{i}}\left[\left(f_{s i}-n_{s i}\right) \frac{Q_{s}^{i}\left(T_{s i}^{p}-T_{p}^{i}\right)}{2}\right] \\
& C_{s}^{i}=\bar{I}_{s i} h_{s i}+b_{s i} \pi_{s i} \\
& C_{s}=\sum_{s=1}^{s} \bar{I}_{s i} h_{s i}+b_{s i} \pi_{s i}
\end{aligned}
$$

\section{Model.}

Based on supply chain in various members of the organization cost assessment calculation, it can be optimized based on the supply chain multi-echelon inventory and distribution mode of integrated inventory and distribution model ( IMLIDM, Integrated Multi-Level Inventory and Distribution Mode ) that the entire supply chain four layer includes the raw material supplier, manufacturer, distribution center and retailer's cost and. Included in the cost of raw materials and finished products inventory holding cost, order cost, setup cost and shortage cost.

$$
\begin{aligned}
& \text { MinSC }=C_{r}+C_{d}+C_{p}+C_{s} \\
& C_{s}=\sum_{s=1}^{s} \bar{I}_{s i} h_{s i}+b_{s i} \pi_{s i} \\
& C_{p}=\bar{I}_{p}\left(h_{p}+C_{p}\right)+\sum_{i=1}^{s} I_{s i}^{p} h_{p i}+A_{p}^{i}\left(f_{s i}-n_{s i}\right) \\
& C_{d}=\sum_{d=1}^{d} A_{d i} Q_{d i} \\
& C_{r}=\sum_{i=1}^{n} C_{r i}=\sum_{i=1}^{n} h_{i r} \bar{I}_{r i}+s_{r i} X_{r i} f_{r i}+b_{r i} \pi_{r i}
\end{aligned}
$$

Constraints

$$
\begin{aligned}
& \hat{T}=L F\left(k, \lambda_{r i} L\right)-\frac{k}{\lambda_{r i}} F\left(k+1 ; \lambda_{r i} L\right) \\
& T_{r i}=\frac{Q+\lambda_{r i} \hat{T}}{\lambda_{r i}} \\
& T_{s}^{i}=k T_{p} \\
& T_{s i}=k^{\prime} T_{s}^{i} \\
& b_{s i}=n_{s i} Q_{s}^{i}
\end{aligned}
$$




\section{Conclusion}

Take distribution quantity and delivery time between members of a supply chain as the main variables, then build integrated vertical multi-stage supply chain inventory structure and distribution model of IMLIDM, that is, the sum cost of four members of the whole supply chain. Cost including raw materials and finished goods inventory holding cost, order cost, production preparation cost and stockout cost. According to the character of the model, using the improved fitness calculation method to design a kind of hybrid simulation annealing method genetic algorithm, used to IMLIDM model optimization. Firstly, use a simple example parameters optimize the model, and analyzed and verified the result, then give investigation data of a company's solar water heater , the second instance substitution model is used to be optimized, finally, we concluded the result of the operation, and reference other scholars model to compare the results. Draw the conclusion that the established IMLIDM model optimization results is better than Integrated Product and Process Development (IPPD) model.

\section{References}

[1] Lu, A.One-vendor multi-buyer integrated inventory model. European Jourrnal of Operational Reasearch. 81(1995)209-210.

[2] Glock, C. H..A multiple-vendor single-buyer integrated inventory model with a variable number of vendors. Computers \& Industrial Engineering, 60(1),173-182. (2011)

[3] Jamal, A. M. M., \& Sarker, B. R.. An optimal batch size for a production system operating under a just-in-time delivery system. International Journal of Production Economics, 32(2), 255-260. (1993)

[4] Nanda, Nam. Quantity discounts using a joint lot size model under learning effects—single buyer case.Computers \& Industrial Engineering, 22(2), 211-221. (1992)

[5] Goyal, S.K. A one-vendor multi-buyer integrated inventory model: a com-ment. European Journal of Operational Research 82, 209-210. (1995)

[6] Goyal, S.K., Nebebe, F.. Determination of economic production-shipment policy for a single-vendor single-buyer system. European Journal of Operational Research 121, 175-178. (2000) 